\title{
The Relationship between Sleep Circadian Rhythm and Central Feedback Mechanism of Gonadal Steroid. in Female Guinea Pigs
}

\author{
SADAO YAMAOKA \\ Department of Physiology, Saitama Medical School \\ 38 Morohongo, Moroyama-cho, Iruma-gun, \\ Saitama, 350-04
}

\begin{abstract}
To examine the relationship between the sleep rhythm and the gonadal feedback system in the guinea pig, the effects of estrous cycle, gonadal steroids and brain deafferentations on the sleep rhythm were studied and the following results were obtained; 1) the guinea pigs did not show an apparent circadian rhythmicity in the sleep-wakefulness cycle but showed an ultradian rhythm, whereas, the activity rhythm was circadian, 2) the rhythm in paradoxical sleep(PS) showed changes associated with the estrous cycle which were characterized by a decrease and rebound-like increase in PS amounts on the day of proestrus, 3) the horizontal deafferentation above the medial preoptic area at the level of the anterior commissure (MPO roof cut) did not disrupt the estrous cycle dependent changes in the PS rhythm, but the prechiasmatic deafferentation of the medial basal hypothalamus (PCD) and the large complete deafferentation of the medial basal hypothalamus (CDL) disrupted them, 4) ovariectomy (OVX) did not result in any changes in sleep and activity rhythms, 5) an administration of estradiol benzoate(E2) to OVX guinea pig caused a decrease in the amount of PS and an administration of progesterone (P) $48 \mathrm{~h}$ after E2 caused a more pronounced decrease and rebound-like increase in the amount of PS, 6) the MPO roof cut did not affect the steroidal modification of the PS rhythm and the PCD disrupted it, while the CDL-animal also showed a E2-induced PS decrease.

From these results, it appears that the guinea pig may be a circadian animal, but this may not be seen in the sleep-wakefulness cycle, and the estrous cycle dependent changes in the PS rhythm may be the reflection of steroidal modification of the sleep rhythm and the site of action may be the inside of the medial preoptic anterior hypothalamic structures, but this area may also be affected by the output from the medial basal hypothalamus.
\end{abstract}

The author $(1978,1980)$ has previously demonstrated that the circadian rhythm of paradoxical sleep (PS) in the female rats showed changes associated with the estrous cycle and the administration of estradiol (E2) with or without progesteron $(\mathrm{P})$ to ovariectomized $(\mathrm{OVX})$ rats resulting in a night PS

\section{Received March 31, 1983.}

This article is dedicated to the memory of Professor M. Kawakami, under whom we had the preasure of studying and working. decrease. These estrous cycle dependent changes and the sex steriodal modification of the circadian PS rhythm seemed to be correlated with the sex steroid feedback mechanisms which might be mediated by the anterior limbic area. On the other hand, the guinea pig is a unique common laboratory rodent having a long estrous cycle with a true luteal phase. In contrast to the rat, the deafferentation of the medial basal hypothalamus $(\mathrm{MBH})$ in the guinea 
pig did not disturb the estrous cycle or ovulation (Terasawa and Wiegend 1978, Butler and Donovan 1971) as in the rhesus monkey (Krei et al., 1975, Norman et al., 1976). However, reports concerning the circadian rhythm in the guinea pig have been quite few and it has not been determined whether the guinea pig is a circadian animal or not.

It was of interest, therefore, to study the species difference in neuroendocrine mechanisms from the viewpoint of the relationship between the circadian sleep rhythm and central feedback mechanisms of gonadal steroid. The present study was undertaken to clarify the correlation of circadian sleep rhythm with the central feedback mechanisms of gonadal steroids.

\section{Materials and Methods}

\section{Animals}

Young adult female guinea pigs (Hartley strain 300 -400g perchased from Tokyo Experimental Animal Corporation) were maintained under controlled humidity $(45-55 \%)$, temperature $\left(24 \pm 1^{\circ} \mathrm{C}\right)$ and lighting conditions (light on 05:00-19:00 h and light off 19:00-06:00 h). Commercial pellets for guinea pig (GM-3, Funabashi Nojo) and tap water were given ad libitum.

The estrous cycle was followed by daily examination (between 09:00-10:00 h) of the vaginal membrane. Vaginal smears were taken by irrigating the vagina with a few drops of clean water on days when the vaginal membrane was spontaneously opened. The smears were observed under a microscope and the cell types present were recorded. The estrous cycle of guinea pigs is regularly 16 to 20 days long. The vaginal membrane spontaneously open for 3-5 days, and thus vaginal smears during vaginal opening were observed. The leucocytic smears are sometimes obtained on 1st day of vaginal opening (late diestrus), the nucleated superficial epitherial cells are characteristic on first or second day of vaginal opening (proestrus), and during the following one or two days vaginal smears contain cornified superficial epitherial cells. The appearance of large numbers of leucocytes is signals the beginning of the next diestrous interval. The animals having regular estrous cycles for 1-2 months were selected for the study.

\section{Experimental groups}

There were 5 experimental group : 1) intact control. 2) operated control, 3) the horizontal deafferentation above the medial preoptic area at the level of the anterior commisure (MPO roof cut), 4) the prechiasmatic deafferentation of the medial basal hypothalamus (PCD), and the large complete deafferentation of the medial basal hypothalamus (CDL). All groups contained 4 to 6 animals.

All animals were ovariectomized after a long term recording experiment for 20 to 30 days. After over one month OVX, all animals were used again for a recording experiment to see the effect of gonadal steroids.

\section{Surgical procedure}

The implantation of electrodes and surgical isolation of neural circuit were performed 5-6 weeks before recording under ether anesthesia. Stereotaxic coordination was based mostly on the atlas by Tindal (1965). But this altas is based on the guinea pig weighing $700-830 \mathrm{~g}$ instead of our animals which weighed $300-430 \mathrm{~g}$. Therefore, we reduced the coordination figure by $5 \%$. The surgical isolation of the neural circuit was preceded by electrode implantation. The locations of various knife cuts are shown in Fig. 1. The MPO roof cut was made with the L-shaped knife ( $3 \mathrm{~mm}$ horizontal blade) at the level of the anterior comissure above the medial preoptic area. The PCD was made with the Halasz' knife ( $3 \mathrm{~mm}$ radius) anteriorly to the suprachiasmatic nucleus. The CDL extending from the caudal border of the optic chiasma to the mammillary bodies was made with the smaller Halasz' knife ( $2 \mathrm{~mm}$ radius).

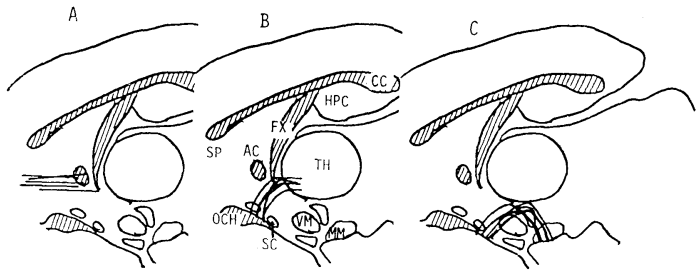

Fig. 1. Midsuggital view of hypothalamic deafferentation. Each solid line indicates the extent of a histologically verified knife cut. A, horizontal deafferentation above the medial preoptic area at the level of anterior commissure (MPO roof cut); $\mathrm{B}$, prechiasmatic deafferentation of medial basal hypothalamus (PCD); C, large complete deafferentation of medial basal hypothalamus (CDL). Abbreviations; $\mathrm{AC}$, anterior commissure; CC, corpus callosum ; FX, fornix ; HPC, hippocampus; MM, mammillary body; $\mathrm{OCH}$, optic chiasma; SC, suprachiasmatic nucleus; SP, septum; VM, ventromedial hypothalamus. 
Bipolar electrodes for EEG recording from the dorsal hippocampus and the pontine reticular formation, the stainless steel screw electrodes for the cortical EEG, and the stainless steel wire electrodes ( $200 \mu \mathrm{m}$ diameter, epoxilite coated) for the dorsal neck EMG and the eye movement were implanted ten days before recording experiments. The electrodes were connected to a small IC connector and were cemented onto the skull. The surgical isolation and electrodes portion were confirmed by postmortem histological examination.

\section{Recording procedure}

Twenty four hrs before recording, two animals were transfered to individual plastic cylindrical cages $(30 \mathrm{~cm}$ in diameter and $40 \mathrm{~cm}$ high) in a sound reduced and electrically shielded room with a silvered mirror for observation. These cages were put on the top pan food balance to record the animal's activity. The shaking of the balance indicator due to the animal's movement was converted to an ONOFF signal by means of a photoelectric switch and counts for every hour were totalled. The IC connector on each animal's head was coupled to a suspended recording cable and the animal was allowed to adapt to the recording conditions for $24 \mathrm{hr}$. The physiological signals for two animals were recorded on a 9 channel EEG recorder with a multipurpose amplifier (ME 95D, Nihon Koden). The polygraphic records at a slow speed $(1 \mathrm{~mm} / \mathrm{sec})$ were divided into 3 sleep-wakefulness stages; alertness(A), slow wave sleep(SWS) and paradoxical sleep(PS), according to the characteristic changes in the cortical, hippocampal and pontine reticular EEGs, the dorsal neck EMG and the eye movements, by visual inspection and with a Sleep Pattern Analyzer (Diamedical System Co.). (Fig. 2)

In all groups, before OVX, the recording was continued for 20-30 days. After OVX, polygraphic recording for 7-10 days was performed. The total duration figures for SWS and PS were expressed for each $2 \mathrm{hr}$ and were shown in a chronogram. The mean amounts of SWS and PS for $12 \mathrm{hr}$ during the same estrous cycle or the same treatment in each

\section{EEG PATTERNS FOR GUINEA PIG}

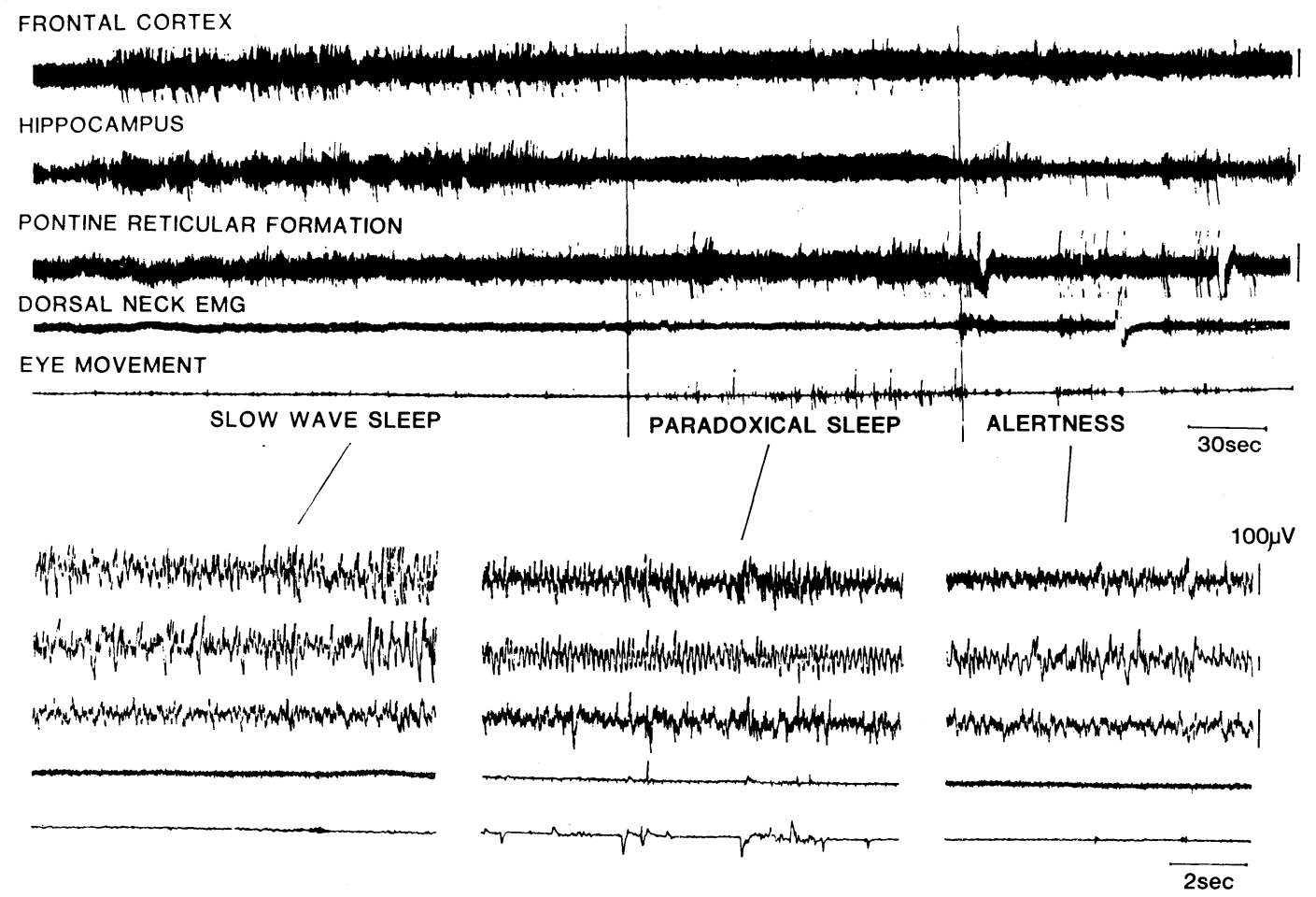

Fig. 2. EEG records from frontal cortex, hippocampus and pontine reticular formation, EMG from dorsal neck muscles and eye movement during slow wave sleep, paradoxical sleep and alertness at slow paper speed $(1 \mathrm{~mm} / \mathrm{sec}$, upper row) and moderate paper speed $(15 \mathrm{~mm} / \mathrm{sec}$, lower row). 
CONTROL

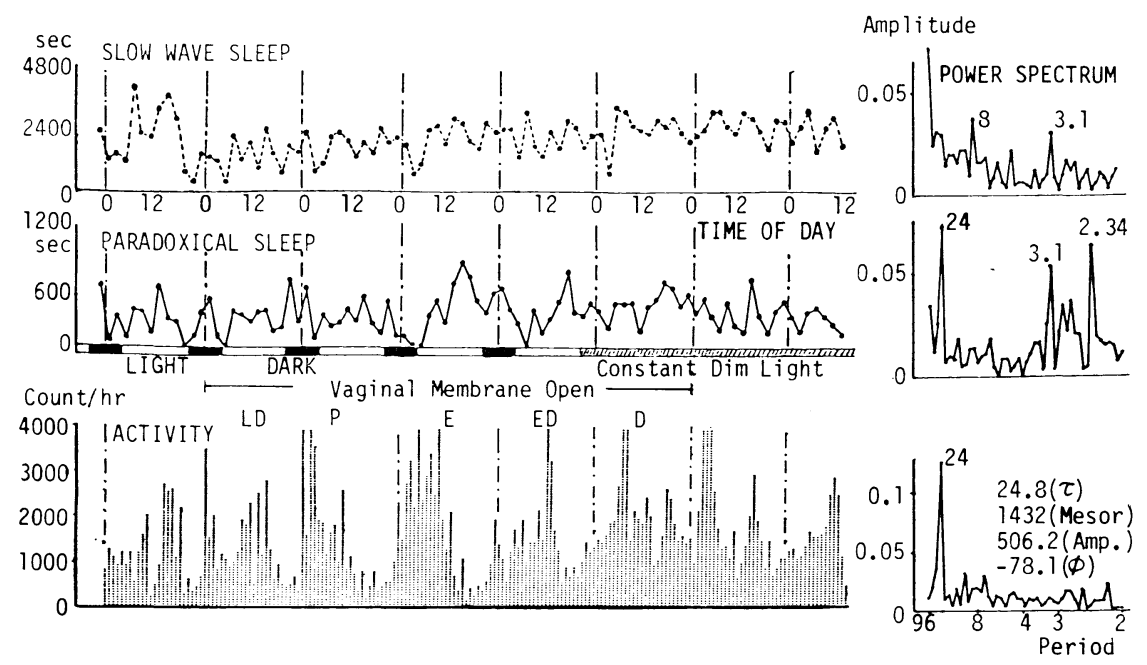

MPO ROOF CUT
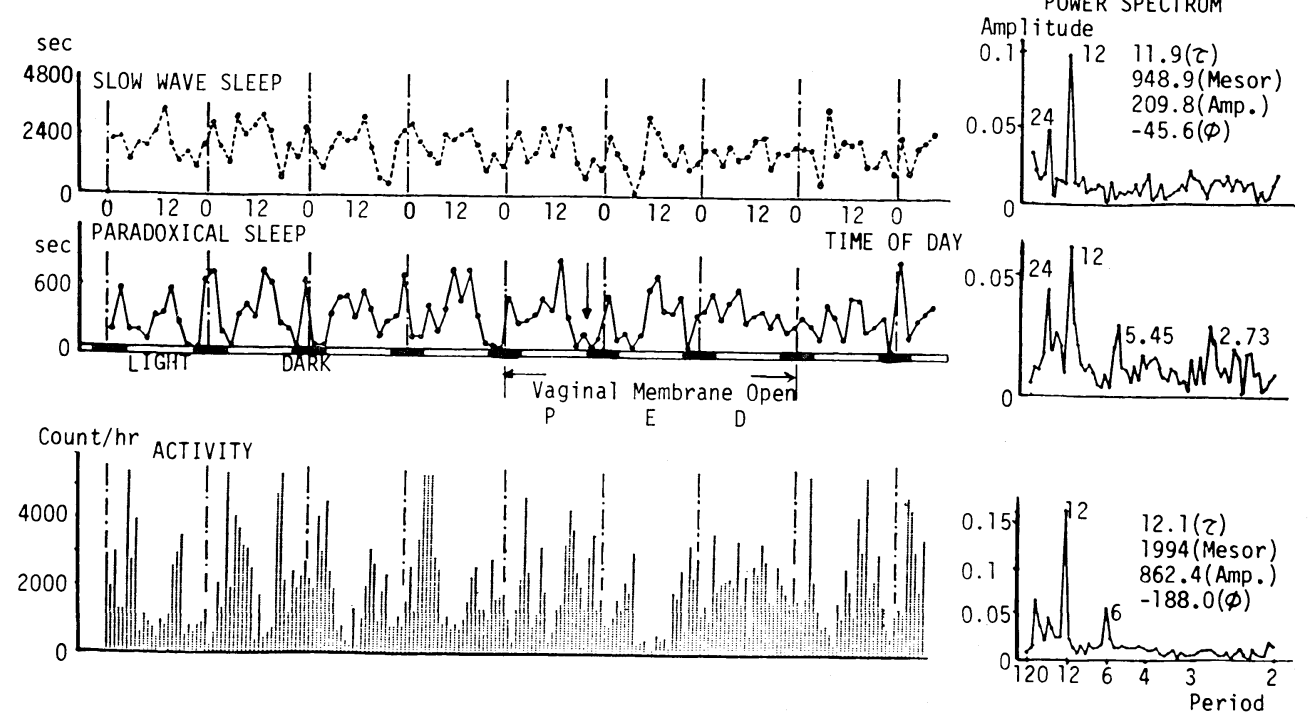

Fig. 3. Bihourly distribution of slow wave sleep $(\bullet \cdots \cdots)$, paradoxical sleep $(\bullet-\bullet)$ and locomoter activity expressed by histogram (counts $/ \mathrm{hr}$ ) under light-dark schedule in an intact female guinea pig and a female guinea pig bearing MPO roof cut. Left graphs indicate the sequencial data of sleep and activity records. Right graphs indicate the power spectral analyses in each record. Vertical axes represent $2 \mathrm{hr}$ amount of slow wave sleep and paradoxical sleep, $1 \mathrm{hr}$ counts of locomoter activity and computed amplitude in power spectral analyses. Horizontal axes represent time of day in left graphs and periods in right graphs. Numbers with peak in right graphs indicate the peak periods (hr) in power spectral analyses. Block data in right graphs indicate the data of least square analyses in dominant peak. Abbreviations: LD, late diestrus; P, proestrus; E, estrus; D, diestrus; Amp., peak amplitude (sec); $\tau$, dominant peak period (hr); $ø$, acrophase (degree). 
group were calculated and were shown in a histogram with standard errors.

\section{Data analyses and statistics}

For each state of sleep, a time series was computed from the sleep duration in each consecutive hour for 7 to 20 consecutive days. This time series was converted to the auto-correlation function which oscillates around the zero axis by subtracting the mean from the original time series. This autocorrelation function was further used in computing the spectral density by means of the harmonic analysis using the Fourier transformation (the power spectral analysis, Sasaki, 1978). The power spectral analysis was used to find the period of the time series for SWS, PS and activity. The least squares method (Sasaki, 1978) was also used to obtain the accurate figures for the period $(\tau)$, mesor and acrophase $(\varnothing)$ of these rhythm components. An electronic computer (IBM 4331 processor) was used in the analysis. The paired Student's t-test was used to find the difference between two mean values for the $12 \mathrm{hr}$ amounts of SWS and PS.

\section{Result}

Rhythmicity of SWS, PS and activity in the control female guinea pig and correlation with estrous cycle.

The SWS and PS rhythm in the female guinea pig showed an ultradian rhythm. The power and least squares spectral analyses showed multiple peaks of amplitude in various periodos $(\tau)$. Their peak periods were distributed from 2.2 to $29.8 \mathrm{hr}$, and the most dominant peak period of amplitude in SWS was around $8 \mathrm{hr}$, but that in PS was different among individual animals. The dominant peak period of PS was around $24 \mathrm{hr}$ in $3,12 \mathrm{hr}$ in $3,8 \mathrm{hr}$ in 2 and $3 \mathrm{hr}$ in one out of 9 animals. On the other hand, the rhythm of running activity showed a semicircadian $(\tau=1.20 \mathrm{hr})$ or a circadian (mean $\tau=26.2 \pm 0.5 \mathrm{hr}$ ) cyclicity. The acrophase of activity was 2:00 a.m. to 6:00 a.m. (Fig. 1.).

All guines pigs in both intact and operated control groups showed a 16-20 day estrous cycle. On the proestrous night, a $12 \mathrm{hr}$ PS amount from 18:00 to $6: 00 \mathrm{hr}$ showed a significant decrease compared with that on other days $(\mathrm{p}<0.01)$ and a $12 \mathrm{hr}$ PS amount from 6:00 to $18: 00 \mathrm{hr}$ on the estrous day was significantly higher than that in the rest of the cycle $(\mathrm{p}<0.01)$. (Table 1$)$.

The guinea pig bearing MPO roof cut and correlation with estrous cycle.

The SWS and PS rhythm in guinea pigs bearing MPO roof cut showed an ultradian or semicircadian rhythm in the bihourly chronogram. In power or least square spectrum analyses of a one hour amount of sleep episode, however, semicircadian components were dominant ( $\tau=12.0 \mathrm{hr}$ ). Running activity showed a circadian rhythmicity (mean $\tau=23.9 \pm 1.0 \mathrm{~h}$ ). The guinea pig bearing MPO roof cut showed a 16-20 day estrous cycle, the same as intact animals. The $12 \mathrm{hr}$ PS appearance from 18:00 to $6: 00 \mathrm{hr}$ on the proestrous night showed a significant decrease compared with that on other days $(\mathrm{p}<0.01)$, but was not followed by a reboundlike PS increase. (Table 1.).

\section{The guinea pig bearing PCD,}

The SWS and PS rhythm in PCD guinea pigs showed an ultradian rhythm in bihourly sleep distribution. (Fig. 4). The power and the least square spectral analyses of hourly sleep episodes showed multiple peaks. The peaks were scattered from 2.1 to $29.9 \mathrm{hr}$. The rhythm of running activity in this group was dominant in the circadian component ( $\tau=20.0-29.0)$ under $24 \mathrm{hr}$ light-dark conditions. The estrous cycle in PCD guinea pigs was completely disrupted and showed persistent vaginal opening. Changes in sleep rhythm associated with the estrous cycle were also disturbed. The $12 \mathrm{hr}$ SWS and PS amount between 6:00-18:00 and 18:0006:00 did not show any significant differences throughout the recording periods.

\section{The guinea pig bearing $C D L$}

The SWS and PS rhythm in CDL guinea pigs showed an ultradian rhythm in bihourly sleep distribution. The power and least 


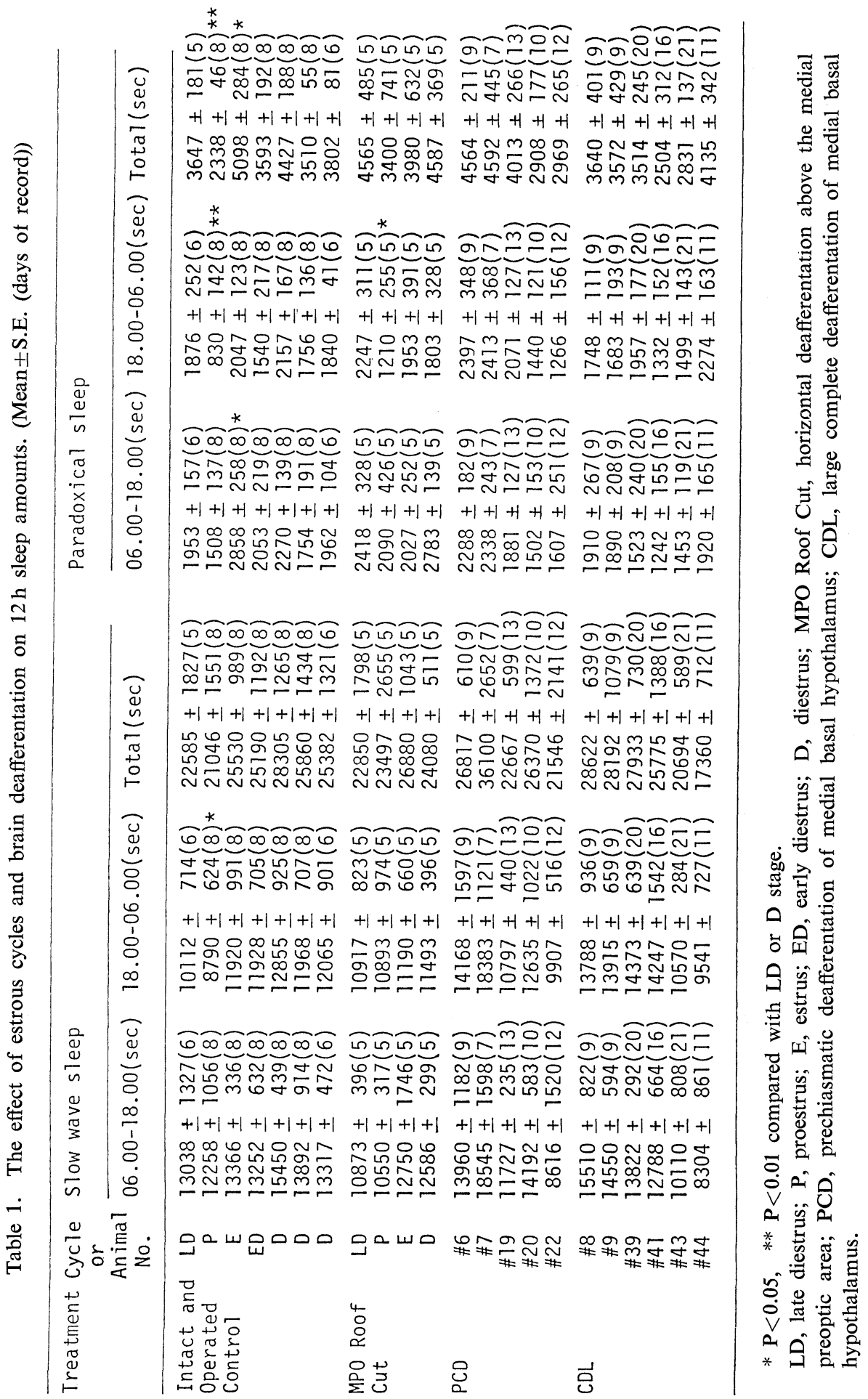




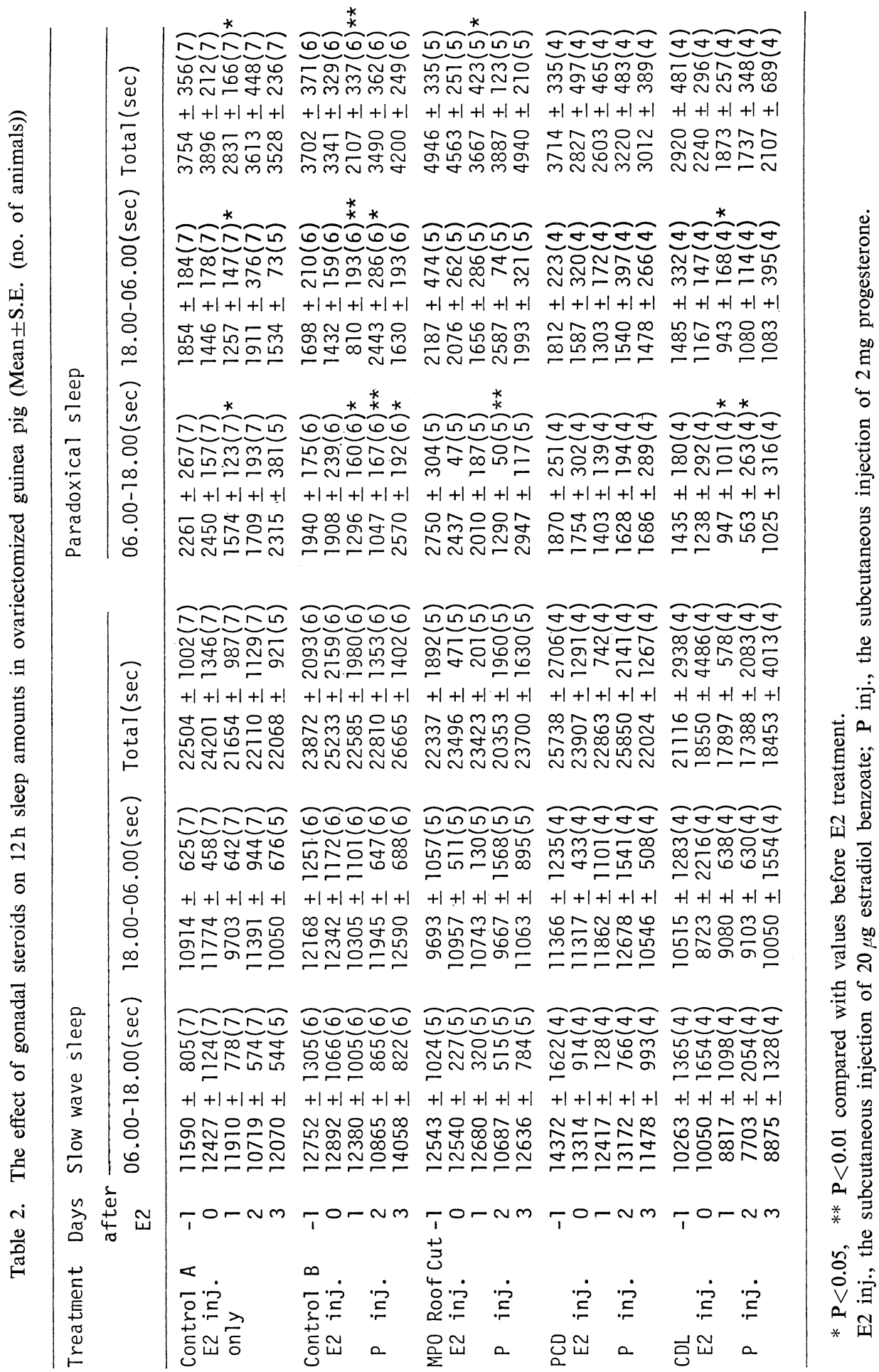



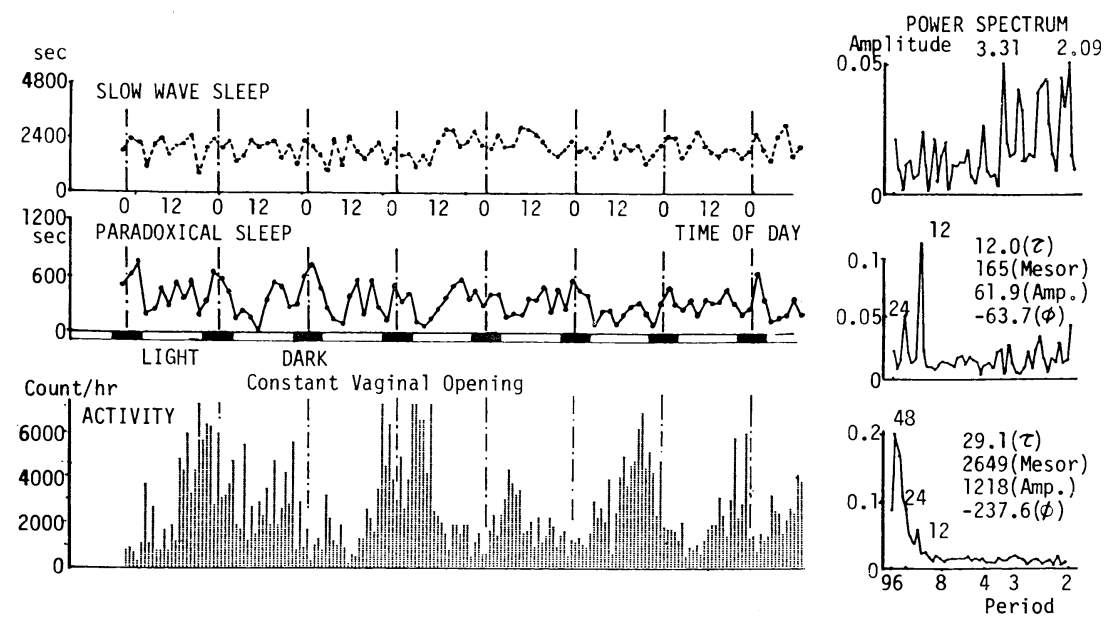

COMPLETE HYPOTHALAMIC DEAFFERENTATION
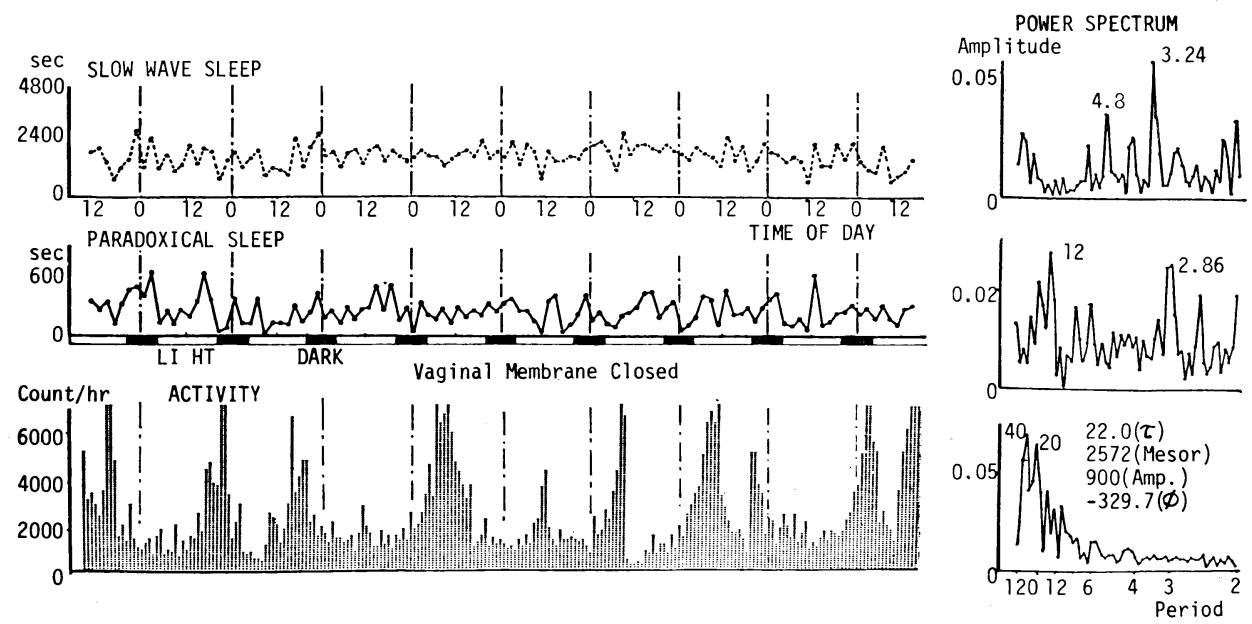

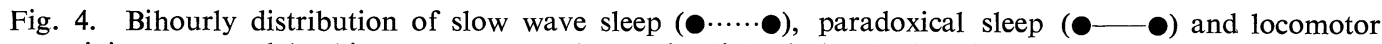
activity expressed by histogram (counts/hr) under light-dark schedule in a female guinea pig bearing prechiasmatic deafferentation and a female guinea pig bearing complete deafferentation. Left graphs indicate the sequencial data of sleep and activity records. Right graphs indicate the power spetral analyses in each record. See Fig. 3. for other information.

square spectral analyses of the one hour sleep amount indicated multiple peaks of amplitude $(\tau=2.3-26.0 \mathrm{hr})$. The dominant peak period different in indivival animals. The rhythm of running activity in CDL guinea pig showed multiple peak periods in power spectral analysis, but still showed the circadian component $(\tau=7.7-25.5 \mathrm{hr})$.
The estrous cycle of this group was different from PCD animals, 2 out of 6 animals showed an irreglar and elongated estrous cycle, and the vaginal membrane opened at 20-40 day intervals. However, no changes in sleep rhythm associated with the estrous cycle were observed during 20 recording days. 
Sleep rhythu of the OVX guinea pig.

The OVX did not change the sleep and activity rhythm in any experimental group but disrupted changes in sleep rhythm associating with the estrous cycle. The power and least square spectral analyses indicated multiple peaks in SWS and PS, but the circadian component in activity rhythm. The peak period of activity rhythm was 20 to $29 \mathrm{hr}$. Twelve hour amounts of SWS and PS also did not observed any difference between 06:00-18:00 hr and 18:00-06:00 hr.

The effect of gonadal steroids on $O V X$ female guinea pig. The intact $O V X$ guinea pig.

The long term OVX guinea pig (one month after OVX) was given a subcutaneous injection of $20 \mu \mathrm{g}$ E2 alone at $12: 00$ on the 3 rd day of polygraphic recording, or $20 \mu \mathrm{g}$ E2 on the 3rd day of recording and $2 \mathrm{mg}$ $P$ at $48 \mathrm{hrs}$ after E2 treatment. The E2 administration gradually decreased the bihourly PS amount beginning 6-8 $\mathrm{hr}$ after E2 treatment, and lasted 1-3 days. The P treatment $48 \mathrm{hr}$ after $\mathrm{E} 2$ injection indicated a more pronounced decrease beginning 6-10 hr after $\mathbf{P}$ and a rebound-like increase beginning $12-14 \mathrm{hr}$ to $24 \mathrm{hr}$ after $\mathrm{P}$ injection. On the other hand, the bihourly SWS amount did not indicate any changes. (Fig. 5 and Table 2). The mean value for $12 \mathrm{hr}$ PS amounts showed a significant decrease following E2 treatment and a significant increase following $P$ treatment, but the mean $12 \mathrm{hr}$ SWS value did not show any significant change following E2 and P treatment.

The OVX guinea pig bearing brain deafferentations

The steroidal effect on sleep rhythm in the OVX-MPO roof cut guinea pig was coincident with that in the intact OVX guinea pig, but that in the PCD-OVX guinea pigs was disrupted.

The E2 administration in the PCD-OVX guinea pig gradually decreased the bihourly
PS amount beginning 20-24 hr after E2 injection, and the $P$ treatment 48 hrs after E2 did not show any changes in E2-induced PS decrease on that day. The decreased PS appearance recovered $20-24 \mathrm{hrs}$ after $\mathrm{P}$ administration. Neither E2 nor $P$ treatment did resulted in any changes in the SWS appearance.

\section{Discussion}

\section{Circadian rhythmicity in guinea pig}

The literature concerned with the circadian rhythm in guinea pigs is very limited. In the present study, the SWS and PS did not show an apparent circadian cyclicity, while the running activity showed a clear circadian cyclicity. Ibuka (1982) reported that immature precocial animals such as the guinea pig, horse or monkey showed the same rhythm characteristics as in adulthood, and guinea pigs showed an ultradian sleep rhythm. But the female cat, an altricial animal, also showed an ultradian rhythm in both the immature and adult period (unpublished data). In the guinea pig, the rhythm of running activity, plasma cortisol contents (Garris, 1979), feeding pattern (Horton et al., 1975), brain serotonin and norepinephrine contents (Philo et al., 1977), histamin response (Sheving et al., 1972), mitotic response (Reeve, 1978), retinal melatonin contents (Pang et al., 1982), and electrical response of pineal cells (Semm and Vollath 1980) showed circadian cyclicity.

It is better to say, therefore, that the guinea pig by nature is a circacian animal, but that this cyclicity did not manifest itself in sleep-wakefulness cycle. Furthermore, it is very interesting that the guinea pig shows three different cycles: 1) the rhythms in the plasma cortisol, brain norepinephrine show a similar pattern to a diurnal animal, 2) the rhythms in brain serotonin, feeding pattern, mitotic response and running activity 


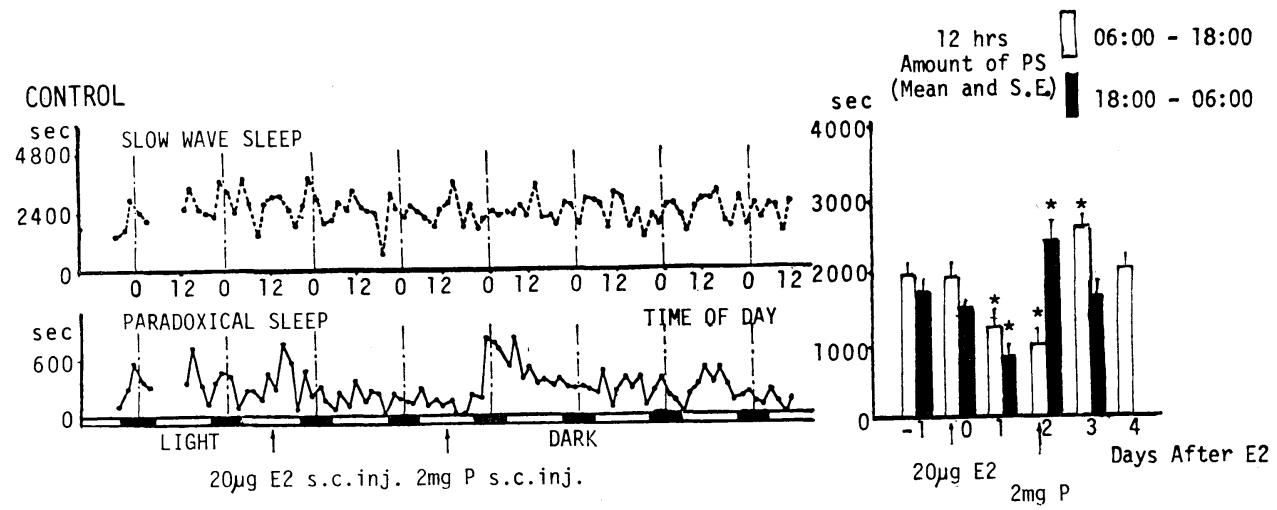

MPO ROOF CUT
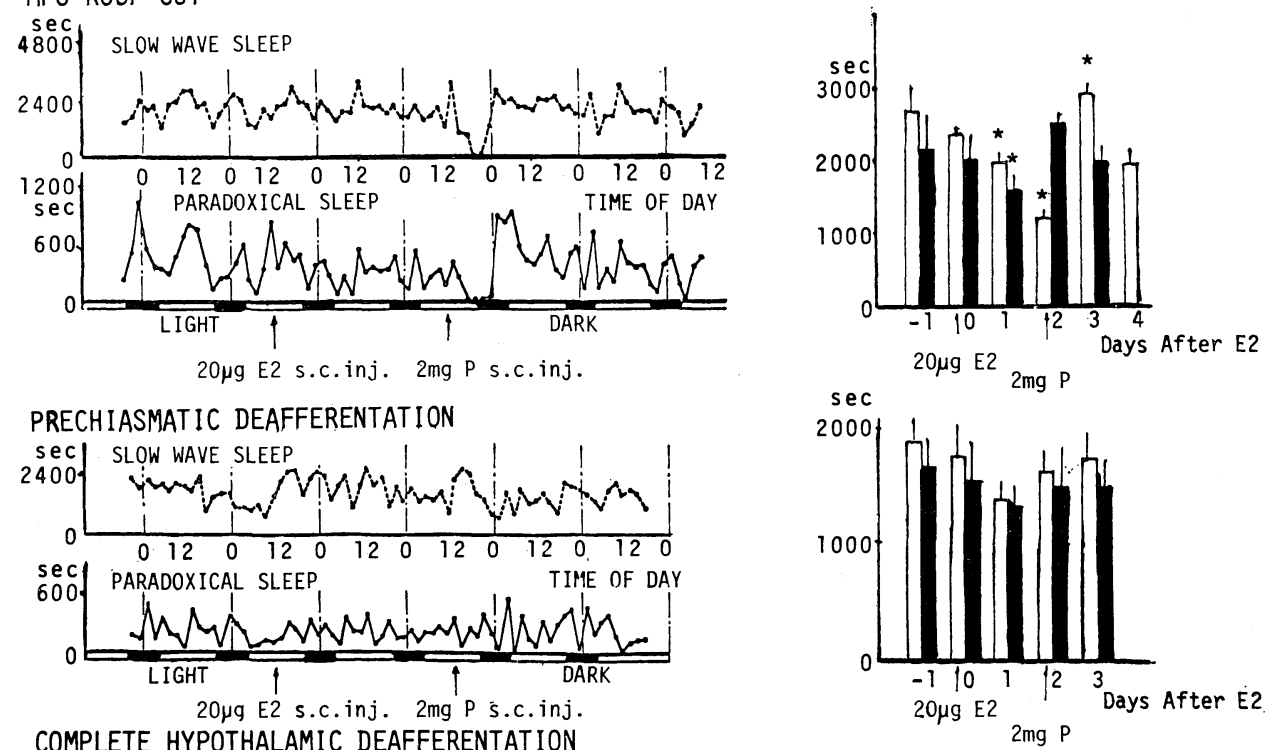

COMPLETE HYPOTHALAMIC DEAFFERENTATION
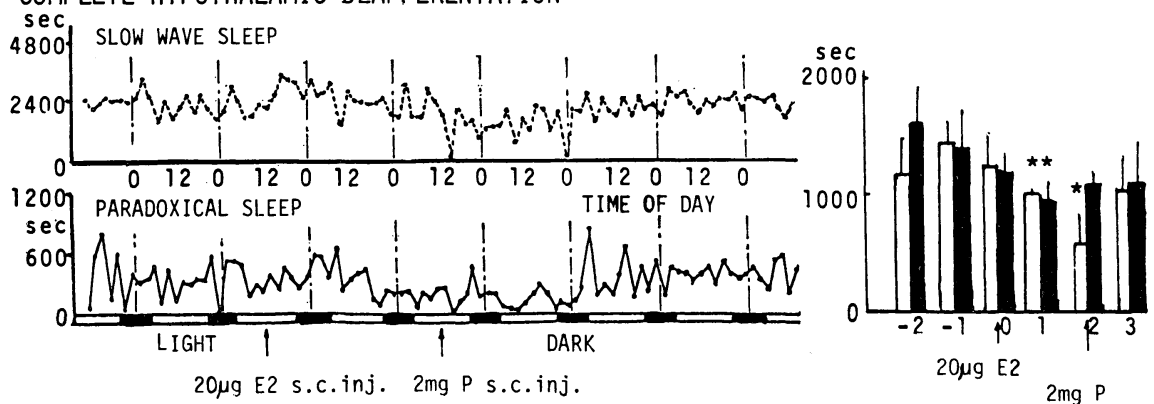

Fig. 5. Effect of gonadal steroids on sleep circadian rhythm in ovariectomized guinea pigs. Left graphs show the sequential data of sleep records. Arrows under each graph of paradoxical sleep represent steroids injection with their dosages. All injections were performed at noon. Right graphs show the $12 \mathrm{hr}$ mean amounts of paradoxical sleep with standard errors for 4-8 guinea pigs during 6.00-18.00 h (open bars) and $18.00-6.00 \mathrm{~h}$ (black bars). E2, estradiol benzoate; P, progesteron; s.c. inj., subcutaneous injection; *, $\mathrm{P}<0.05$. 
show a similar pattern to a nocturnal animal and 3 ) the rhythm of sleep-wakefulness and testosteron (Pajot and Delost 1977) indicate an ultradian cyclicity. The reason for these various results in the rhythmicity of guinea pig cannot be explained at this point.

\section{Sleep rhythm and estrous cycle}

The author (1980) has previously reported that the PS rhythm in the female rats reflected the steroid feedback activity. The estrous cycle in the guinea pig is normally 16-20 days long with a true luteal phase. The present study demonstrated that the guinea pig also has changes in PS rhythm dependent in the estrous cycle, and this phenomenon is maintained after MPO roof cut, but disrupted after PCD. These reuslts resembled those for rats. Terasawa and Wiegend (1978) reported that the CDL-guinea pig maintained the normal estrous cycle and ovulation. In the present study, 2 out of $6 \mathrm{CDL}$-guinea pigs showed the estrous cycle (periods were 18-25 days), but during 20 days recording period, the vaginal opening was not observed. Thus, it was not clear whether the CDL guinea pig shows the estrous cycle dependent PS changes or not.

\section{Sleep rhythm and sex steroid}

The author has demonstrated the following results for the rat: 1) The OVX caused a splitting of circadian PS rhythm and increased appearance of night PS. 2) $20 \mu \mathrm{g}$ E2 treatment indicated a night PS decrease beginning on the day of E2 treatment and lasting 5-7 days regardless of the second E2 or $\mathrm{P}$ treatment $72 \mathrm{hr}$ after the first E2. 3) This E2 effect reflected the positive feedback condition of E2.

In the present study, 1) the OVX guinea pig sleep and activity rhythm did not change, 2) the $20 \mu \mathrm{g}$ E2 treatment induced a PS decrease, but did not change ultradian cyclicity, 3) the $\mathbf{P}$ treatment $48 \mathrm{hr}$ after E2 caused a further decrease in PS which was followed by a rebound-like increase begin- ning $10-12 \mathrm{hr}$ after the $P$. Malven and Sawyer (1966) had reported similar results, but their recording period was only $8 \mathrm{hr}$ per day. Since Terasawa et al. (1979) showed in the guinea pig that the LH surge occurred $36 \mathrm{hr}$ after the E2 treatment and 6 hrs after the $P$ treatment $(30 \mathrm{hr}$ after the E2), these PS changes would not depend on the LH changes in blood due to sex steroids, but would be due to the direct effect of sex steroids in the central nervous system.

The MPO roof cut in rats disrupted the E2 induced night PS decrease, but the guinea pig with the MPO roof cut showed both the E2 induced and P induced PS decreases as well as the rebound-like PS increase. The PCD disrupted the steroid induced PS changes. This result seems to indicate that the site of the steroidal modification of sleep rhythm in the guinea pig is inside the medial basal hypothalamus (MBH) and the medial preoptic and anterior hypothalamic (MPO$\mathrm{AH})$ neuroendocrine input to the $\mathrm{MBH}$ may affect the gonadotropin release and may modify the sleep rhythm. However, the CDL-OVX guinea pig showed a PS decrease after the E2 treatment, but did not show a PS increase after $P$ treatment. Terasawa and Wiegend (1978) obtained similar results When checking ovulation and gonadotropin release. They interpreted them to mean that there may exist an MPO-AH neuroendocrine connection to the $\mathrm{MBH}$ or the CDL may differentialy isolate normally counterbalancing facilitatory and inhibitory input to neural elements within the $\mathrm{MBH}$ regulating the ovulatory release of gonadotropins. The meaning of our results on the CDLOVX guinea pig may be different from those of Terasawa and Wiegend, since the steroidal modification of sleep rhythm is due to the neural mechanism while ovulation is due to the gonadotropin secretion.

The following hypothesis may be offered to reconcile these contradictory results: 1) the gonadotropins released from the pituitary 
in CDL guinea pig may influence the outside of the MBH and modulate the PS rhythm. 2) the CDL may isolate the output from $\mathrm{MBH}$ which influences the steroidal modification of sleep rhythm and may alter the neural element outside the $\mathrm{MBH}$ to directly effect the steroidal modification of the sleep rhythm.

Regarding the first hypothesis, the present study revealed that the E2 induced PS decrease did not depend on the gonadotropin release. Therefore, this hypothesis could be neglected. As to the second hypothesis, the MPO roof cut did not disrupt the E2 and $P$ modification of PS rhythm and the PCD disrupts the E2 and $\mathrm{P}$ modification. Therefore, the neural connection from the MPO$\mathrm{AH}$ to $\mathrm{MBH}$ plays an important role in the steroidal modification of PS rhythm. However, the CDL induced a PS decrease after the E2 treatment, suggesting that the MPO$\mathrm{AH}$ independently affects the neural mechanism for steroidal modification of PS rhythm.

From these results it may be concluded that 1) the guinea pig may be a circadian animal, but this is not rnanifested in the sleep-wakefulness rhythm, 2) the estrous cycle dependent changes in PS rhythm may be a reflection of the modification of PS rhythm by the gonadal steroid, 3) the main site of this steroidal modification in the guinea pig may be the outside of the $\mathrm{MBH}$ (probably the MPO-AH area), but this site is strongly affected by the $\mathrm{MBH}$, but not by the limbic area in contrast to the rat.

\section{Acknowledgements}

The author is grateful to Dr. K. Katsuura, Department of Physics, Saitama Medical School and Mr. T. Onodera for the programming of computer analyses. The author also thanks Messrs. T. Onodera and H. Masuda for their technical assistanse.

This investigation was supported by a Grant-inAid for Scientific Research (No. 448109) and a Grantin-Aid for Special Project Research (No. 57123116) from The Ministry of Education, Science and Culture of Japan.

\section{References}

Ibuka, N. (1982). The control mechanism of sleepwakefulness rhythms. Protein, Nucleic Acid and Enzyme 27, 246-258. (In Japanese).

Butler, J. E. M. and B. T. Donovan (1971). The effect of surgical isolation of the hypothalamus upon reproductive function in the female guinea pig. J. Endocr. 50, 507.

Garris, D. R. (1979). Diurnal fluctuation of plasma cortisol levels in the guinea pig. Acta Endocrinol. 90, 692-695.

Horton, B. J., C. E. West and S. D. Turley (1975). Diurnal variation in the feeding pattern of guinea pigs. Nutr. Metabol. 18, 294-301.

Krey, L. C., W. R. Butler and E. Knobil (1975). Surgical disconnection of the medial basal hypothalamus and pituitary function in the rhesus monkey. I. Gonadotropin secretion. Endocrinology 96, 1073-1087.

Malven, P. V. and C. H. Sawyer (1966). Sleep patterns in female guinea pigs; effect of sex hormones. Exptl. Neurol. 15, 229-239.

Norman, R. L., J. A. Resko and H. G. Spies (1976). The anterior hypothalamus: how it affects gonadotropin secretion in the rhesus monkey. Endocrinology 99, 59-71.

Pajot, par J. and P. Delost (1977). Teneurs plasmatiques et testiculaires en androgenes au cours du nycthemeres chez le cobaye a la puberte. $C$. $R$. Soc. Biol. 171, 1240-1245.

Pang, S. F., H. S. Yu and P. L. Tang (1982). Regulation of melatonin in the retinae of guinea pigs: effect of environmental lighting. J. Exptl. Zool. 222, 11-15.

Philo, R., P. K. Rudeen and R. J. Reiter (1977). A comparison of the circadian rhythms and concentrations of serotonin and norepinephrine in the telencephalon of four rodent species. Comp. Biochem. Physiol. 57, 127-130.

Reeve, D. R. E. (1978). The diurnal variation of mitotic response in the epithelium at the edge of acute ulcers in the rectal mucous membrane of guinea-pigs. J. Anat. 125, 337-348.

Sasaki, T. The search of the periodic components. In: Jikanseibutsugaku (Chronobiology) (edited by T. Sasaki and Y. Chiba) Asakura Shoten, Tokyo, p. 312-332. (1978). (In Japanese)

Scheving, L. E., G. S. Sohal, C. D. Enna and J. E. Pauly (1973). The persistence of a circadian rhythm in histamine response in guinea pigs maintained under continuous illumination. Anat. Rec. 175, 1-6.

Semm, P. and L. Vollath (1980). Electrophysiological evidence for circadian rhythmicity in mammalian pineal organ. J. Neural Transm. 47, 181190. 
Terasawa, E., J. S. Rodrigues, W. E. Bridson and S. J. Wiegend (1979). Factors influencing the positive feedback action of estrogen upon the luteinizing hormone surge in the ovariectomized guinea pig. Endocrinology 104, 680-686.

Terasawa, E. and S. J. Wiegend (1978). Effects of hypothalamic deafferentation on ovulation and estrous cyclicity in the female guinea pig. Neuroendocrinology 26, 229-686.

Tindal, J. S. (1965). J. Comp. Neur. The forebrain of the guinea pig in stereotaxic coordinates. 124, 259-266.

Yamaoka, S. (1978). Participation of limbic-hypothalamic structures in circadian rhythm of slow wave sleep and paradoxical sleep in the rat. Brain Res. 151, 255-268.

Yamaoka, S. (1980). Modification of circadian sleep rhythms by gonadal steroids and the neural mechnisms involved. Brain Res. 185. 385-398. 